Ciencias Agrarias / Agricultural Sciences

Cienc Tecn UTEQ (2020) 13(2) p 1-8 ISSN 1390-4051; e-ISSN 1390-4043 (c) (i) () () ()

doi: https://doi.org/10.18779/cyt.v13i2.386

\title{
Patrones de uso de la tierra en la Amazonía central ecuatoriana: Una comparación entre etnias Kichwa, Shuar y colonos
}

\section{Land use patterns in the central Ecuadorian Amazon: A comparison between ethnic groups Kichwa, Shuar and colonos}

\author{
Cristian Vasco ${ }^{1}$, Shirley Huerta Cruz $^{2}$, María Isabel Jaramillo ${ }^{3}$, Rusbel Jaramillo ${ }^{1}$, Estefanía Jácome ${ }^{1}$ \\ ${ }^{1}$ Facultad de Ciencias Agrícolas, Universidad Central del Ecuador, Quito, Ecuador; clvasco@uce.edu.ec \\ ${ }^{2}$ Facultad de Ciencias Administrativas, Universidad de Guayaquil, Guayaquil, Ecuador \\ ${ }^{3}$ Instituto Superior Tecnológico Ismael Pérez Pazmiño, Machala, Ecuador
}

Rec.: 10.02.2020. Acept.: 29.05.2020. Publicado el 30 de diciembre de 2020

\section{Resumen}

$\mathrm{E}_{\mathrm{p} \text { s }}^{\mathrm{s}}$ ste artículo analiza los patrones de uso de la tierra de poblaciones colono-mestizas e indígenas (Kichwa y Shuar) en la Amazonía ecuatoriana. Con datos de una encuesta de hogar realizada en comunidades colonas, Kichwa y Shuar de la provincia de Pastaza, Ecuador, se determinó que los colonos se dedican principalmente a la agricultura comercial y a la ganadería extensiva. Por otra parte, existen diferencias marcadas en los patrones de uso de la tierra de las dos etnias indígenas estudiadas. Como los colonos, los Shuar se dedican a la producción de cultivos comerciales y la crianza de ganado vacuno, en tanto que los Kichwa se dedican casi exclusivamente a la agricultura de subsistencia. Estas diferencias parecen estar relacionadas con las condiciones locales, trayectorias de migración y asentamiento y el grado de integración a la economía de mercado. Más allá de esto, los resultados de este estudio indican que, contrariamente a la creencia general, las estrategias de supervivencia y los patrones de uso de la tierra de los colonos no necesariamente ni en todos los casos difieren de aquellas adoptadas por las poblaciones indígenas.

Palabras clave: uso del suelo-colonos-pueblos indigenas-Amazonía-Ecuador

\begin{abstract}
This paper analyzes the land use patterns of mestizo-colonists and indigenous populations in the Ecuadorian Amazon. With data from a household survey conducted in colonist, Kichwa and Shuar communities in the Pastaza province, Ecuador, we determined that, as expected, colonists mostly engage in commercial agriculture and cattle ranching. There are substantial differences in land use patterns of both indigenous groups under study. Like colonists, the Shuar engage in produce cash crops and cattle, whereas the Kichwa almost exclusively engage in subsistence farming. Such different patterns appear to be related to local conditions, migratory and settlement patterns and the degree of exposure to the market economy. Beyond this, the results presented here indicate that, contrary to the general wisdom, the livelihood strategies and land use patterns of indigenous peoples do not necessarily differ from those adopted by indigenous peoples.
\end{abstract}

Keywords: land use-colonist-indigenous peoplesAmazon-Ecuador 


\section{Introducción}

$\mathrm{L}$ a Amazonía ecuatoriana es considerada una de las áreas más biodiversas del planeta y por tanto una de las prioridades de conservación a nivel global (Myers et al., 2000). Sin embargo, en las últimas décadas, esta zona ha experimentado uno de los procesos de deforestación más acelerados en la cuenca amazónica. De entre las varias actividades humanas que afectan al ambiente, la expansión agrícola es señalada como la principal causa de la deforestación tropical (Briassoulis, 2000). Por tanto, el estudio de los patrones uso de la tierra en la región amazónica sigue siendo una prioridad para el diseño de políticas encaminadas a conciliar el desarrollo rural y la conservación.

Los estudios previos sobre uso de la tierra en la Amazonía hacen una clara distinción entre los patrones de uso de la tierra de los colonos que migraron a la Amazonía a partir de los sesenta y aquellos de los pueblos indígenas amazónicos. Los patrones agrícolas de los colonos se caracterizan por: su escaso nivel de tecnificación, la continua incorporación de áreas de bosque a la producción agrícola; y como consecuencia de lo anterior, su, incompatibilidad con el uso sostenible de recursos naturales (Murphy et al., 1997; Pichón, 1997). Por el contrario, los patrones de uso del suelo de las poblaciones indígenas son generalmente asociados con prácticas agrícolas sustentables y de poco impacto sobre el medio ambiente (e.g., agricultura de subsistencia y largos períodos de descanso de las parcelas) (Dufour, 1990; Schwartzman et al., 2000). Sin embargo, varios estudios dan cuenta que los indígenas también incursionan en actividades económicas poco sustentables, una vez que han entrado en contacto con los colonos y la economía de mercado (Gray et al., 2008; Lu et al., 2009).

Los cambios en los patrones de uso de la tierra son una causa de preocupación entre quienes promueven la conservación de los bosques amazónicos dadas: la importancia de los territorios indígenas para la conservación de los bosques tropicales (Davis y Wali, 1994; Nepstad et al., 1999), las altas tasas de deforestación en territorios controlados por colonos (FAO, 2005), las altas tasas de crecimiento poblacional dentro de las poblaciones indígenas (Gray et al., 2008) y el acelerado proceso de integración de las poblaciones indígenas a la economía de mercado (Lu, 2007).

Con datos de una encuesta de hogar llevada cabo entre mayo y noviembre de 2013, este estudio compara los patrones de uso del suelo entre poblaciones colonas e indígenas (Kichwa y Shuar) de la provincia de Pastaza. El resto de este artículo se organiza de la siguiente manera: la primera sección pone en contexto las poblaciones Kichwa, Shuar y colonas de la Amazonía ecuatoriana, posteriormente se describe el área de estudio, la encuesta y el proceso de recolección de datos. En la sección subsiguiente se reportan y discuten los resultados, mientras que la sección final presenta las conclusiones y algunos comentarios finales.

\section{Los colonos, los Kichwa y los Shuar de la Amazonía ecuatoriana}

Durante la década de los sesenta, luego del descubrimiento de reservas petroleras en la Amazonía y la apertura de carreteras para la construcción de oleoductos y pozos petroleros, oleadas de campesinos empobrecidos, originarios principalmente de la región andina, migraron a la Amazonía en busca de tierras (Bilsborrow et al., 2004). Aunque este fue principalmente un proceso de migración espontánea, también fue estimulada desde el gobierno como una manera de ocupar e incorporar a la producción agrícola lo que, hasta ese entonces se consideraban "las tierras baldías" del Oriente (Martínez Novo, 2012; Pástor Pazmiño y Alvarado, 2012). Dichos migrantes trajeron consigo patrones agrícolas caracterizados por bajos niveles de tecnificación, alta dependencia de mano de obra familiar y una marcada inclinación por la ganadería extensiva (Pichón, 1997). Como consecuencia, durante los noventa, las tasas de deforestación en la Amazonía ecuatoriana llegaron a estar entre las más altas del mundo (Torres et al., 2014).

En las últimas décadas, la presión sobre los recursos naturales se ha intensificado como consecuencia de las altas tasas de natalidad de la población colona y el subsiguiente proceso de subdivisión de fincas, lo que ha convertido a la tierra en un recurso que dejó de ser abundante (Bilsborrow et al., 2004). La población de colonos en la provincia de Pastaza asciende a 46,000 habitantes, quienes están asentados en la parte más occidental de la provincia, en las inmediaciones de Puyo (Figura 1). Los colonos asentados en Pastaza se especializan en la producción de cultivos comerciales como naranjilla (Solanum quitoense), papa china (Colocasia esculenta) y caña de azúcar (Saccharum officinarum), además de la crianza extensiva de ganado vacuno. (Prefectura de Pastaza, 2012).

Los Kichwa Canelos son los habitantes tradicionales de la provincia de Pastaza. Su número asciende a aproximadamente 18,000 individuos (INEC, 2010). Varios estudios (Guzmán-Gallegos, 1997; Uzendoski, 2004) describen que los Kichwa amazónicos se dedican principalmente a la agricultura de subsistencia, principalmente yuca (Manihot esculenta) y plátano (Musa sp.), la recolección de productos del bosque, la caza y la pesca, en niveles sustentables y con poco impacto sobre el medio ambiente. Sin embargo, otros estudios describen que los Kichwa, principalmente 
aquellos asentados cerca de carreteras y centros poblados, están cada vez más integrados a la economía de mercado y han adoptado las formas de producción y subsistencia de los colonos, incluyendo la agricultura orientada al mercado, la ganadería y la explotación de madera (Mejía et al., 2015; Vasco et al., 2015). Los Kichwa controlan alrededor de 1'400,000 ha en Pastaza bajo un esquema de derechos de usufructo. Si bien las densidades poblacionales en comunidades Kichwa son relativamente bajas, Sirén (2007) argumenta que, en el futuro próximo, pueden existir problemas de escasez de tierra agrícola en comunidades con densidades poblacionales relativamente altas.

Los Shuar son los habitantes tradicionales de la Amazonía Sur de Ecuador, principalmente de la provincia de Morona Santiago, donde reside el 80\% de la población Shuar del Ecuador (INEC, 2010). Durante los sesenta y setenta, como una estrategia para contrarrestar el asentamiento de colonos en sus territorios, los Shuar reclamaron derechos ancestrales comunitarios sobre extensas áreas de bosque en Morona Santiago con el apoyo de ONGs católicas (Rudel et al., 2002). Para este efecto, los Shuar siguieron la misma estrategia utilizada por los colonos en aquella época, es decir, tumbar una porción de bosque, plantar pastos y establecer pequeños rebaños de ganado (Rudel et al., 2002). Esta estrategia fue utilizada no solo en sus territorios nativos sino también en las provincias hacia el Norte (Pastaza, Napo y Orellana), donde han establecido comunidades y han incursionado en actividades como la agricultura comercial, la ganadería, la explotación de madera y el empleo no agrícola (Bremner y Lu, 2006).

La población Shuar de Pastaza asciende a 5600 individuos quienes se asientan mayoritariamente a lo largo de la vía hacia Morona Santiago (INEC, 2010). Aunque los Shuar de Pastaza controlan alrededor de 200,000 ha bajo esquemas de propiedad comunitaria (Prefectura de Pastaza, 2012), las densidades poblacionales en comunidades Shuar son mayores que las observadas en comunidades Kichwa (Vasco y Sirén, 2016), lo que podría tener importantes implicaciones en el uso y conservación de recursos naturales.

\section{Materiales y métodos}

\section{Área de estudio y encuesta}

Pastaza es la provincia más extensa pero a la vez la menos poblada de Ecuador. La parte occidental, donde se concentran los centros urbanos, está poblada principalmente por colonos mientras que la parte oriental es habitada fundamentalmente por nacionalidades indígenas, las cuales representan el $60 \%$ de la población rural de Pastaza (INEC, 2010), siendo las más numerosas las etnias Kichwa y Shuar. Los colonos, principalmente originarios de la serranía central de Ecuador, se especializan en cultivos comerciales como naranjilla, papa china y caña de azúcar, además de la crianza extensiva de ganado. Las nacionalidades indígenas, en tanto, tienen derechos ancestrales comunitarios sobre extensos territorios y normalmente ganan su sustento

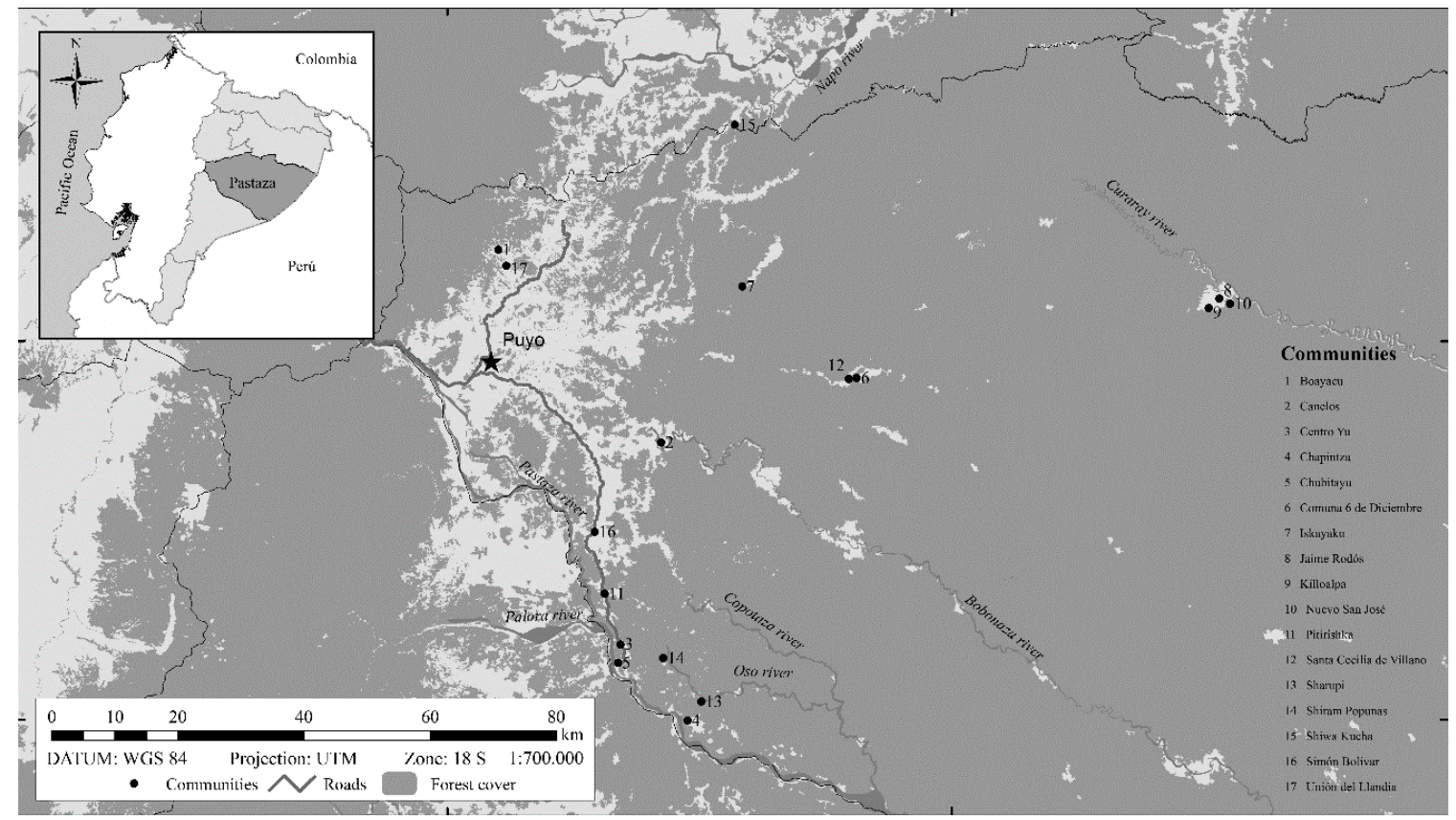

Figura 1. Área de estudio en la provincia Pastaza. 
Cuadro 1. Comunidades en la muestra.

\begin{tabular}{|c|c|c|c|c|}
\hline Comunidad & Población & $\begin{array}{l}\text { Grupo étnico } \\
\text { predominante }\end{array}$ & Medio de acceso & $\begin{array}{c}\text { Tiempo al Puyo } \\
\text { (horas) }\end{array}$ \\
\hline Comuna 6 de Diciembre & 45 & Colonos & $\begin{array}{c}\text { Carretera de } \\
\text { tierra }\end{array}$ & 3.5 \\
\hline Boayacu & 50 & Colonos & $\begin{array}{l}\text { Carretera de } \\
\text { tierra }\end{array}$ & 1.0 \\
\hline Unión del Llandia & 180 & Colonos & $\begin{array}{c}\text { Carretera de } \\
\text { tierra }\end{array}$ & 0.5 \\
\hline Simón Bolívar & 3000 & Colonos & $\begin{array}{c}\text { Carretera } \\
\text { pavimentada }\end{array}$ & 0.5 \\
\hline Centro Yu & 50 & Shuar & $\begin{array}{c}\text { Carretera de } \\
\text { tierra }\end{array}$ & 1.5 \\
\hline Shiram Popunas & 141 & Shuar & Sendero & 6.0 \\
\hline Sharupi & 94 & Shuar & Sendero & 3.0 \\
\hline Chapintsa & 420 & Shuar & $\begin{array}{l}\text { Carretera de } \\
\text { tierra }\end{array}$ & 2.0 \\
\hline Pitirishka & 250 & Shuar & $\begin{array}{c}\text { Carretera } \\
\text { pavimentada }\end{array}$ & 0.75 \\
\hline Chubitayu & 1125 & Shuar & $\begin{array}{c}\text { Carretera } \\
\text { pavimentada }\end{array}$ & 1.0 \\
\hline Iskayaku & 60 & Kichwa & Sendero & 3.0 \\
\hline Shiwa Kucha & 310 & Kichwa & $\begin{array}{l}\text { Carretera de } \\
\text { tierra }\end{array}$ & 2.0 \\
\hline Jaime Roldós & 75 & Kichwa & Río & 8.0 \\
\hline Killoalpa & 75 & Kichwa & Río & 8.0 \\
\hline Nuevo San José & 150 & Kichwa & Río & 8.0 \\
\hline Santa Cecilia & 150 & Kichwa & $\begin{array}{l}\text { Carretera de } \\
\text { tierra }\end{array}$ & 3.5 \\
\hline Canelos & 1200 & Kichwa & $\begin{array}{l}\text { Carretera de } \\
\text { tierra }\end{array}$ & 1.0 \\
\hline
\end{tabular}

de la agricultura de subsistencia (plátano y yuca) y la recolección de productos del bosque, caza y pesca (Prefectura de Pastaza, 2012). Sin embargo, en las últimas décadas los indígenas también han incursionado en la agricultura orientada al mercado y la extracción de madera con fines comerciales (Macdonald et al., 1993).

Los datos utilizados provienen de encuestas de hogar y comunidad llevadas a cabo entre mayo y noviembre de 2013. Las encuestas se elaboraron con base en la metodología PEN (Poverty and Environment Network) para bosques tropicales (CIFOR, 2007). El cuestionario se diseñó para obtener información demográfica, sobre el uso del suelo, uso de recursos naturales, ingresos (agrícolas y no agrícolas) y bienes del hogar. Para la recolección de datos se utilizó un muestreo en dos etapas. En la primera etapa se seleccionaron 17 comunidades siguiendo criterios de accesibilidad, infraestructura y etnia (ver Cavendish, 2003). En la segunda etapa se seleccionaron hogares aleatoriamente dentro de cada comunidad. En total se encuestaron 4 comunidades mestizas, 6 comunidades Shuar y 7 comunidades Kichwa (Cuadro 1) para un total de 304 hogares distribuidos de la siguiente manera: 116 hogares Kichwa, 120 hogares Shuar y 68 hogares colonos. El procedimiento utilizado y la muestra obtenida aseguran una buena representación de la diversidad de realidades y grados de desarrollo de Pastaza.

\section{Resultados y discusión}

1 analizar los patrones de uso de suelo en la
Amazonía ecuatoriana es importante tener en
cuenta las características socioeconómicas de los
hogares. El Cuadro 2 presenta estadísticas de algunas
características socioeconómicas que pueden tener
alguna influencia sobre los patrones agrícolas de los
hogares rurales amazónicos.
Los jefes de hogar Shuar son mayores y mejor 
educados que los jefes de hogar Kichwa y colonos. Los hogares Kichwa son más numerosos que los Shuar y colonos. Una posible explicación para este fenómeno es que la migración a hacia áreas urbanas, otras provincias e incluso hacia otros países son estrategias de supervivencia comunes en las poblaciones Shuar y colonas (Rudel et al., 2002). Estudios previos (Vasco et al., 2015; Vasco et al., 2013) dan cuenta que el empleo fuera de finca es en la actualidad una de las principales fuentes de ingresos en la Amazonía ecuatoriana. Los datos aquí presentados son consistentes con este enunciado ya que, en promedio, $79 \%$ de todos los hogares encuestados reciben ingresos del trabajo fuera de finca. El porcentaje de hogares con ingreso fuera de finca es menor en la etnia Kichwa, lo que podría estar relacionado con que los hogares Kichwa se asientan en áreas alejadas de las carreteras y del Puyo (ver datos de distancia a carretera y distancia al Puyo), lo cual reduce las oportunidades de empleo no agrícola (Vasco y Bislborrow, 2016).

Más de la mitad de los hogares Shuar ha recibido un crédito durante los 12 meses previos a la realización de la encuesta. Una posible explicación para este resultado es que los Shuar exhiben un marcado interés en la ganadería (Gray et al., 2008; Rudel et al., 2002), actividad que demanda fuertes inversiones iniciales (compra de ganado). Pese a que evidencia previa (Gray et al., 2008; Lu et al., 2009) sugiere que los Shuar están más integrados a la economía de mercado que los Kichwa, los resultados de este estudio indican que los hogares Shuar asisten casi al doble de reuniones comunitarias (22) que sus pares Kichwa (12).
Estudios previos sostienen que el "ciclo de vida del hogar", o el tiempo de residencia de un hogar en un determinado lugar, tiene incidencia directa sobre los patrones de uso de la tierra (Pan y Bilsborrow, 2005; Pichón, 1997). De acuerdo a este postulado, los mayores niveles de conversión de bosque a usos agrícolas ocurren al inicio del asentamiento. En este estudio el tiempo de residencia se dividió en: corto ( $\leq 10$ años), medio (10-25 años) y largo (>25 años). Los porcentajes de hogares con permanencia corta, media y larga son casi equivalente para hogares colonos y Shuar, sin embargo, existen marcadas diferencias con respecto a los hogares Kichwa. El porcentaje de hogares Kichwa con residencia larga (79\%) dobla al de colonos (38\%) y Shuar $(42 \%)$ en esta categoría.

En contraparte, el porcentaje de hogares Kichwa con residencia corta es la tercera parte de los valores reportados para colonos y Shuar. Aunque los Shuar son originarios del Sur de la Amazonía, durante los setenta y ochenta muchos de ellos migraron hacia el norte presionados por las altas tasas de crecimientos poblacional y el colapso del cultivo de naranjilla en sus territorios de origen (Rudel et al., 2002); factores similares a los que motivaron a empobrecidos campesinos andinos a migrar a la Amazonía durante los sesenta.

Una cuarta parte de los hogares colonos y el 14\% de los hogares Shuar usan insumos agrícolas (fertilizantes químicos y pesticidas). Una posible explicación para este resultado es que estos dos grupos étnicos han incursionado en la agricultura orientada al mercado, la cual a diferencia de la agricultura de subsistencia, hace

Cuadro 2. Características socioeconómicas por grupo étnico.

\begin{tabular}{|c|c|c|c|c|}
\hline Variable & $\begin{array}{c}\text { Promedio } \\
\text { general }\end{array}$ & Colonos & Kichwa & Shuar \\
\hline Edad del jefe de hogar & 41.6 & 45.4 & 41.5 & 39.7 \\
\hline Educación del jefe de hogar (años) & 8.3 & 8.2 & 7.5 & 9.6 \\
\hline Tamaño del hogar & 4.5 & 3.7 & 5.3 & 4.2 \\
\hline Porcentaje de hogares con empleo no agrícola & 79 & 85 & 64 & 81 \\
\hline Porcentaje de hogares que han recibido crédito & 36 & 44 & 21 & 56 \\
\hline Distancia a la carretera más cercana $(\mathrm{km})$ & 15.00 & 2.08 & 25.59 & 1.74 \\
\hline Tiempo del viaje al Puyo (minutos) & 154 & 45 & 283 & 135 \\
\hline Asistencia a reuniones comunitarias & 14 & 7 & 11 & 22 \\
\hline Porcentaje de hogares con residencia $\leq 10$ años & 18 & 26 & 7 & 24 \\
\hline Porcentaje de hogares con residencia $10-25$ años & 25 & 36 & 14 & 33 \\
\hline Porcentaje de hogares con residencia $>25$ años & 55 & 38 & 79 & 42 \\
\hline Porcentaje de hogares que usan insumos agrícolas & 12 & 24 & 4 & 14 \\
\hline Densidad poblacional (habitantes $/ \mathrm{km}^{2}$ ) & 17.57 & 60.68 & 6.35 & 11.58 \\
\hline Tenencia de la tierra & & Privada & \multicolumn{2}{|c|}{ Comunitaria } \\
\hline
\end{tabular}


uso intensivo de insumos externos a la finca. Finalmente, al comparar las densidades poblacionales a nivel de comunidad, los datos indican que las comunidades colonas presentan valores hasta diez veces más altos que los observados en comunidades indígenas.

Una variable importante en las decisiones sobre uso de la tierra es el tipo de tenencia de la tierra: privada vs. comunitaria. En el caso de este estudio, esta variable está estrechamente ligada a la etnicidad dado que todos los hogares colonos tienen derechos privados sobre la tierra, mientras que los indígenas poseen derechos comunitarios de usufructo, es decir pueden disponer de la tierra que les ha sido asignada por la máxima autoridad (asamblea comunitaria) para fines agrícolas más no pueden venderla (Bremner y Lu, 2006; Gray et al., 2008).

El Cuadro 3 muestra el área agrícola total, así como las áreas promedio dedicadas a cultivos de subsistencia (plátano y yuca), cultivos comerciales (naranjilla) y pastos para cada grupo étnico. El área total disponible para los hogares Kichwa (64 ha) es considerablemente más alta que aquellas reportadas para hogares Shuar (45 ha) y colonos (27 ha). Estas cifras pueden estar relacionadas con que las densidades poblacionales son considerablemente menores es en comunidades Kichwa. Los resultados también muestran que los Shuar mantienen áreas agrícolas mayores que las observadas para hogares colonos y Kichwa, siendo la diferencia de más del doble con respecto a estos últimos. El área dedicada a cultivos de subsistencia (plátano y yuca) difiere ligeramente entre hogares Kichwa y Shuar, pero es considerablemente menor para los colonos. Una posible explicación para este hallazgo es que los colonos pueden adquirir estos productos en mercados locales, mientras que los indígenas, quienes habitan mayoritariamente en zonas alejadas de los centros poblados, dependen en gran medida de lo que puedan cultivar en sus parcelas.

Es importante analizar el área dedicada al cultivo de naranjilla debido principalmente a dos razones: i) este es el principal cultivo comercial y por tanto una de las principales fuentes de ingresos para los habitantes de esta zona, y ii) el cultivo de naranjilla está estrechamente ligado a la deforestación debido que es altamente susceptible al ataque de nemátodos y requiere, por tanto, de suelos recientemente desmontados, ricos en materia orgánica y libres de nemátodos (INIAP, 2009).

Alrededor del 28, 37 y 7\% de los hogares colonos, Shuar y Kichwa, respectivamente, producen naranjilla, lo cual demuestra la importancia de este cultivo, especialmente para hogares colonos y Shuar. La parcela promedio de los productores colonos y Shuar (1.2 ha) dobla a la de los productores Kichwa (0.6 ha). Cerca de $32 \%$ de los hogares en la muestra (43\% de colonos, $10 \%$ Kichwa y $55 \%$ Shuar) reportan tener pastizales. El área promedio en pastizales de los hogares Kichwa es la mitad de la registrada para los hogares Shuar y la tercera parte de la de los hogares colonos.

Estas cifras son consistentes con literatura previa (Bremner y Lu, 2006; Gray et al., 2008; Lu et al., 2012) que identifica a los Shuar como la etnia indígena amazónica más integrada a los mercados agrícolas y la que más ha adoptado los patrones de uso de la tierra observados en los colonos. Si bien es cierto, en términos relativos, los Shuar dedican menos tierra (19\% del total disponible) a fines agrícolas comparados con sus pares colonos (23\% del total disponible), estas cifras muestran que, en la actualidad, una proporción importante de los hogares Shuar se han especializado en la agricultura comercial, lo cual tiene importantes implicaciones ambientales, dado que como se mencionó anteriormente, la agricultura es la principal causa de deforestación en la Amazonía. En contraparte, los hogares Kichwa dedican una ínfima parte de su tierra (4\% del total disponible) a usos agrícolas, principalmente de subsistencia. Estos hallazgos son consistentes con los de Lu et al. (2009), quienes concluyen que existen grandes diferencias en los patrones de uso de la tierra entre las distintas nacionalidades indígenas de la Amazonía ecuatoriana.

Cuadro 3. Patrones de uso agrícola de la tierra por tipo de cultivo y etnia.

\begin{tabular}{lcccc}
\hline \multicolumn{1}{c}{ Variable } & $\begin{array}{c}\text { Promedio } \\
\text { general }\end{array}$ & Colonos & Kichwa & Shuar \\
\hline Área total (ha) & 50.33 & 27.76 & 64.03 & 45.25 \\
Área total cultivada (ha) & 5.77 & 6.55 & 3.12 & 8.57 \\
Cultivos de subsistencia (plátano y yuca) (ha) & 1.41 & 0.61 & 1.60 & 1.84 \\
Cultivos comerciales (naranjilla) (ha) ${ }^{a}$ & 1.10 & 1.20 & 0.56 & 1.21 \\
Pastos (ha) $^{\text {a }}$ & 10.50 & 15.20 & 5.13 & 9.20 \\
\hline
\end{tabular}

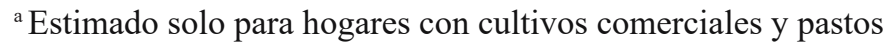




\section{Conclusiones y consideraciones finales}

L os resultados aquí presentados son consistentes con estudios previos que indican que hay variaciones significativas en los patrones de uso de la tierra entre las nacionalidades indígenas amazónicas ( $\mathrm{Lu}$ et al., 2009), siendo la nacionalidad Shuar la que más se ha integrado a la economía de mercado y más ha adoptado los patrones de uso de la tierra que caracterizan a los colonos (Bremner y Lu, 2006; Gray et al., 2008; Lu et al., 2012). Por otro lado, los hallazgos de este estudio están en contraposición con literatura previa que, como regla general, asume que las prácticas agrícolas de los indígenas tienen un bajo impacto ambiental y son compatibles con el medio ambiente (Dufour, 1990; Nepstad et al., 1999; Schwartzman et al., 2000). Con un sistema vial más extenso, mayor acceso a educación formal, crédito y tecnologías agrícolas; y mayores tasas de crecimiento poblacional, esta premisa parece no aplicar a todos los casos.

Es importante reflexionar sobre las similitudes en los patrones de asentamiento y características socioeconómicas de los Shuar y los colonos. Los Shuar comparten varias de las características observadas en los colonos en el pasado. Como los colonos, los Shuar migraron hacia Pastaza como respuesta a las altas tasas de crecimiento poblacional y la escasez de recursos en sus comunidades de origen. De manera similar a los colonos, los Shuar se asentaron en las inmediaciones de carreteras. En términos de fuentes de ingreso, como los colonos, los Shuar han diversificado sus fuentes de ingresos, recurren a créditos formales, usan insumos agrícolas y han incursionado en la agricultura comercial y la ganadería. Con estos antecedentes, no es sorpresivo que los patrones de uso del suelo de los Shuar sean bastante similares a los observados en los colonos.

La adopción de patrones de uso de la tierra propios de los colonos y el uso poco sostenible de recursos naturales no son exclusivos de los Shuar. Por ejemplo, Gray et al. (2008) describen el caso de los Secoya de la Amazonía Norte, quienes a través del tiempo se han especializado en la producción pecuaria gracias al apoyo financiero y la asistencia técnica de ONGs y empresas petroleras. También en la Amazonía Norte, Loaiza et al. (2015) dan cuenta que los Kichwa que residen cerca de carreteras obtienen la mayor parte de sus ingresos de la extracción de madera. Estos antecedentes junto con los resultados de este estudio sugieren que diferentes grupos étnicos exhiben diferentes estrategias de supervivencia y diferentes patrones de uso de la tierra dependiendo de: sus patrones migratorios y de asentamiento, las condiciones locales específicas, el grado de exposición a la economía de mercado y la presencia de agentes externos (e.g., ONGs y empresas petroleras/mineras).

Dada la similitud de estos grupos con los colonos en términos de patrones de uso del suelo y estrategias de supervivencia, las políticas encaminadas a promover la conservación del bosque en estas áreas demandan de intervenciones similares a las utilizadas en territorios de colonos (pago por servicios ambientales, creación de fuentes de ingresos alternativas a la agricultura, agricultura sustentable, entre otras).

El caso de los Kichwa es diferente ya que: en su mayoría nacieron en las comunidades en que residen, tienen menos acceso al empleo no agrícola y al crédito formal y residen en comunidades localizadas en áreas apartadas de centros poblados y con menor densidad poblacional. Posiblemente debido a estas características, los Kichwa se dedican fundamentalmente a la agricultura de subsistencia y solo de manera marginal a la agricultura comercial y la ganadería. Si bien es cierto, estos resultados sugieren que no se requieren mayores intervenciones de conservación en este tipo de comunidades, las mismas podrían ser necesarias en el futuro debido al crecimiento poblacional y el acelerado proceso de integración a la economía de mercado de los pueblos indígenas en general (Lu, 2001; Sirén, 2007).

\section{Literatura citada}

Bilsborrow, Richard, Alison Barbieri y William Pan. (2004). "Changes in Population and Land Use Over Time in the Ecuadorian Amazon". Acta Amazonica, No. 4, Vol. 34, pp. 635-647.

Bremner, Jason y Flora Lu. (2006). "Common Property among Indigenous Peoples of the Ecuadorian Amazon". Conservation and Society, No. 4, Vol. 4, pp. 499-521.

Briassoulis, Helen (2000). "Analysis of land use change: theoretical and modeling approaches"; En The Web Book of Regional Science, pp. 110-120, Loveridge, S, editores. Morgantown: West Virginia University.

Davis, Shelton y Alaka Wali. (1994). "Indigenous Land Tenure and Tropical Forest Management in Latin America". No. 8, Vol. 23, pp. 485-490.

Dufour, Darna. (1990). "Use of Tropical Rainforests by Native Amazonians". Bioscience, No. 9, Vol. 40, pp. 652-659.

FAO. (2005) 'State of the World's Forests 2005'. Rome: United Nations Food and Agricultural Organization.

Gray, Clark, Richard Bilsborrow, Jason Bremner y Flora Lu. (2008). "Indigenous Land Use in the Ecuadorian Amazon: A Cross-cultural and Multilevel Analysis". Human Ecology, No. Vol. 36, pp. 97-109.

Guzmán-Gallegos (1997). Para que la Yuca Beba nuestra Sangre. Quito: Abya-Yala.

INEC (2010) Censo de Población y Vivienda 2010. Quito, Ecuador. 
INIAP. (2009) 'INIAP QUITOENSE-2009. Naranjilla de jugo (Solanum quitoense Lam.) injerta en patrones de solanáceas silvestres resistentes a Fusarium ixysporum y a Meloidogyne incognita'. Quito, Ecuador: INIAP.

Loaiza, Toa, Ute Nehren y Gerard Gerold. (2015). "REDD+ and incentives: An analysis of income generation in forest-depe ndent comm unities of the Yasuní Biosphere Rese rve". Applied Geography, No. Vol. 62, pp. 225-236.

Lu, Flora. (2001). "The Common Property Regime of the Huaorani Indians of Ecuador: Implications and Challenges to Conservation". Human Ecology, No. 4, Vol. 29, pp. 425-447.

Lu, Flora. (2007). "Integration into the Market among Indigenous Peoples: A Cross - Cultural Perspective from the Ecuadorian Amazon.”. Current Anthropology, No. 4, Vol. 48, pp. 593-602.

Lu, Flora, Richard E. Bilsborrow y Ana Isabel Ona (2012). Modos de Vivir y Sobrevivir: Un Estudio Transcultural de Cinco Etnias en la Amazonia Ecuatoriana. Quito, Ecuador: ABYA-YALA.

Lu, Flora, Clark Gray, Richard Bilsborrow, Carlos Mena, Christine Erlien, Jason Bremner, Alison Barbieri y Stephen Walsh. (2009). "Contrasting Colonist and Indigenous Impacts on Amazonian Forests". Conservation Biology, No. 3, Vol. 24, pp. 881-885.

Macdonald, Theodore, Dominique Irvine y L. Esther Aranda (1993). "The Quicua of Eastern Ecuador"; En Indigenous Views of Land and the Environment, pp. Davis, S H, editores.

Martínez Novo, Rodrigo. (2012). "Del tiempo insostenible y del sentido del tiempo en las comunidades Kichwa canelos". Desacatos, No. Vol. 40, pp. 111-126.

Mejía, Elena, Pablo Pacheco, Ayme Muzo y Bolier Torres. (2015). "Smallholders and timber extraction in the Ecuadorian Amazon: amidst market opportunities and regulatory constraints". International Forestry Review, No. S1, Vol. 17, pp. 38-50.

Murphy, Laura, Richard Bilsborrow y Francisco Pichón. (1997). "Poverty and prosperity among migrant settlers in the Amazon rainforest frontier of Ecuador". The Journal of Development Studies, No. 2, Vol. 34, pp. 3566.

Myers, Norman, Russell Mittermeier, Cristina Mittermeier, Gustavo Fonseca y Jennifer Kents. (2000). "Biodiversity hotspots for conservation priorities". Nature, No. Vol. 403, pp. 853-858.

Nepstad, Daniel, Adalberto Verssimo, Ane Alencar, Carlos Nobre, Eirivelthon Lima, Paul Lefebvre1, Peter Schlesinger, Christopher Potter, Paulo Moutinho, Elsa Mendoza, Mark Cochrane y Vanessa Brooks. (1999). "Large-scale impoverishment of Amazonian forests by logging and fire". Nature, No. Vol. 398, pp. 505-508.

Pan, William K.Y. y Richard E. Bilsborrow. (2005). "The use of a multilevel statistical model to analyze factors influencing land use: a study of the Ecuadorian Amazon". Global and Planetary Chang, No. Vol. 47, pp. 232-252.

Pástor Pazmiño, C y Marcela Alvarado (2012). Napo y Pastaza: derecho al territorio frente a la colonización y el extractivismo. Quito, Ecuador: SIPAE.

Pichón, Francisco. (1997). "Colonist Land-Allocation Decisions, Land Use, and Deforestation in the Ecuadorian Amazon Frontier". Economic Development and Cultural Change, No. 4, Vol. 45, pp. 707-744.

Prefectura de Pastaza. (2012) 'Plan de Desarrollo de la Provincia de Pastaza al año 2025 '. Puyo, Ecuador: Prefectura de Pastaza.

Rudel, Thomas, Diane Bates y Rafael Machinguiashi. (2002). "Ecologically Noble Amerindians? Cattle Ranching and Cash Cropping among Shuar and Colonists in Ecuador". Latin American Research Review, No. 1, Vol. 37, pp. 144-159.

Schwartzman, Stephan, Adriana Moreira y Daniel Nepstad. (2000). "Rethinking Tropical Forest Conservation: Perils in Parks". Conservation Biology, No. 5, Vol. 14, pp. 1351-1357.

Sirén, Anders. (2007). "Population Growth and Land Use Intensification in a Subsistence-ba sed Indigen ous Community in the Amazon". Human Ecology, No. Vol. 39, pp. 669-680.

Torres, Bolier, Richard Bilsborrow, Alisson Barbieri y Alexandra Torres. (2014). "Changes in income strategies of rural households in the Northern Ecuadorian Amazon". Revista Amazónica- Ciencia y Tecnología, No. 3, Vol. 3, pp. 221-257.

Uzendoski, Michael. (2004). "Manioc Beer and Meat: Value, Reproduction and Cosmic Substance among the Napo Runa of the Ecuadorian Amazon". The Journal of the Royal Anthropological Institute, No. 4, Vol. 10, pp. 883-902.

Vasco, Cristian, Richard Bilsborrow y Bolier Torres. (2015). "Income diversification of migrant colonists vs. indigenous populations: Contrasting strategies in the Amazon". Journal of Rural Studies, No. Vol. 42, pp. $1-10$.

Vasco, Cristian y Richard Bislborrow. (2016). "Aporte del empleo fuera de finca los de ingresos en hogares indígenas y mestizos de la Amazonía ecuatoriana". Economía Agrícola y Recursos Naturales, No. 1, Vol. 16, pp. 5-18.

Vasco, Cristian, B Herrera, S Vargas y R Arias. (2013). "Empleo Agrícola y no Agrícola en la Amazonía Ecuatoriana". Ecuador Debate, No. 3, Vol. 90, pp. 141151.

Vasco, Cristian y Anders Sirén. (2016). "Correlates of wildlife hunting in indigenous communities in the Pastaza province, Ecuadorian Amazonia”. Animal Conservation. 